\title{
BICONTINUOUS LINEAR TRANSFORMATIONS IN CERTAIN VECTOR SPACES*
}

\author{
E. R. LORCH
}

A bounded linear transformation $T$ of a vector space $\mathfrak{B}$ into itself will be called bicontinuous if the inverse of $T$ (defined, of course, over the entire space) exists and is continuous. The group of bicontinuous transformations is dominant in any study of equivalence of transformations; that is, in the determination of the conditions implying relations of the type $B=T^{-1} A T$. The results of this paper constitute an analysis of the structure of bicontinuous transformations in certain spaces. Previous results of the author $\dagger$ indicate that the analysis here given is of a nature which will be useful in the equivalence problem, not only for transformations $A$ which have a simple spectrum but also in more general situations.

First the notion of a basis in a normal linear vector space $\mathfrak{B} \ddagger$ is considered. A sequence of vectors $\left\{\phi_{n}\right\}, \phi_{n} \varepsilon \mathfrak{B}$, is customarily said to form a basis for $\mathfrak{B}$ (necessarily separable) if for every $f \mathfrak{\varepsilon} \mathfrak{B}$ there exists a unique sequence of numbers $\left\{a_{n}\right\}$ such that $f=\sum_{1}^{\infty} a_{n} \phi_{n}$. This definition is open to two objections: It involves the notion of order of the elements of the basis; and for that reason it does not permit of immediate extension to nonseparable spaces. A new definition of basis is given below which is free of these objections. Proceeding in what is apparently another direction, the notion of complete orthonormal set in Hilbert space (these sets form bases) may be extended to spaces $\mathfrak{B}$. Thus after modifying suitably the idea of orthogonality and normality, one obtains a "heterogonal set." It is proved below that the concepts of heterogonal set and of basis are equivalent.

In the second part, we deal exclusively with complex euclidean spaces $\& ; \|$ briefly, these spaces are either unitary spaces, Hilbert spaces, or nonseparable spaces of the Hilbert space type. The principal result is that if $\left\{\phi_{\alpha}\right\}$ and $\left\{\psi_{\alpha}\right\}$ are bases (or heterogonal sets) in $\mathbb{E}$, then there exists a bicontinuous transformation $T$ such that $T \phi_{\alpha}=\psi_{\alpha}$. Since the $T$ transform of a basis is a basis (see below), the

* Presented to the Society, September 6,1938, under the title Bicontinuous linear transformations in complex euclidean spaces.

$\dagger$ On a calculus of operators in reflexive vector spaces, Transactions of this Society, vol. 45 (1939), pp. 217-234.

¥ S. Banach, Théorie des Opérations Linéaires, p. 53.

§ J. Schauder, Mathematische Zeitschrift, vol. 26 (1927), pp. 47-65.

|| Löwig, Acta Litterarum ac Scientiarum, Szeged, vol. 7 (1934), pp. 1-33. 
characterization of bicontinuous transformations from this angle is complete.

1. Bases and heterogonal sets in $\mathfrak{B}$. We first introduce a definition.

Definition 1. A set of elements $\left\{\phi_{\alpha} ; \alpha \varepsilon M\right\}$ with $\phi_{\alpha} \varepsilon \mathfrak{B}$ will be said to be a basis in $\mathfrak{B}$ if

(i) $K^{-1} \leqq\left\|\phi_{\alpha}\right\| \leqq K$, for some number $K$, for all $\alpha \varepsilon M$;

(ii) To every $f \in \mathfrak{B}$, there is associated a unique set of numbers $\left\{a_{\alpha} ; \alpha \varepsilon M\right\}$. The association is such that $a_{\alpha}=0$ except for at most a denumerable set of $\alpha \varepsilon M$ and $f=\sum_{\alpha \in M} a_{\alpha} \phi_{\alpha}$, the convergence to the element $f$ being independent of the order of summation.

A set of vectors $\left\{\psi_{\alpha} ; \alpha \varepsilon M\right\}$ is said to be an independent set if $\sum_{1}^{n} a_{\alpha_{i}} \psi_{\alpha_{i}}=0$ implies $a_{\alpha_{i}}=0, i=1, \cdots, n$. The set is said to span $\mathscr{B}$ if the elements $\sum_{1}^{n} a_{\alpha_{i}} \psi_{\alpha_{i}}$ lie dense in $\mathfrak{B}$. A set $\left\{\gamma_{\alpha} ; \alpha \varepsilon M\right\}$ with $\gamma_{\alpha}=0$ or 1 will be called a set of selectors. Clearly, $\left\{\left(1-\gamma_{\alpha}\right) ; \alpha \varepsilon M\right\}$ is then also a set of selectors. A subset of $\left\{\psi_{\alpha} ; \alpha \varepsilon M\right\}$ (to which the element $0 \varepsilon \mathfrak{B}$ may have been added) may be indicated by $\left\{\gamma_{\alpha} \psi_{\alpha} ; \alpha \varepsilon M\right\}$.

A projection $P$ in $\mathfrak{B}$ is a bounded linear transformation such that $P^{2}=P$. With such a transformation are associated two closed linear manifolds $\mathfrak{M}$ and $\mathfrak{N}$ having the properties $P=I$ (the identity) on $\mathfrak{M}$; $P=0$ on $\mathfrak{N}$; every element $f \varepsilon \mathfrak{B}$ is uniquely expressible in the form $f=g+h, g \varepsilon \mathfrak{M}, h \varepsilon \mathfrak{R}$. A converse statement may be made. Let $\left\{\gamma_{\alpha} ; \alpha \varepsilon M\right\}$ be a set of selectors, let $\mathfrak{M}$ be the closed linear manifold spanned by $\left\{\gamma_{\alpha} \psi_{\alpha} ; \alpha \varepsilon M\right\}$, let $\mathfrak{N}$ be that spanned by $\left\{\left(1-\gamma_{\alpha}\right) \psi_{\alpha} ; \alpha \varepsilon M\right\}$. If for every $f \varepsilon \mathfrak{B}$, the equation $f=g+h$ with $g \varepsilon \mathfrak{M}, h \varepsilon \mathfrak{N}$ has a unique solution, then $\mathfrak{M}$ and $\mathfrak{N}$ determine a projection in the sense indicated above. All projections subsequently treated are of this type.

Definition 2. $A$ set $\left\{\psi_{\alpha} ; \alpha \varepsilon M\right\}$ with $\psi_{\alpha} \varepsilon \mathfrak{B}$ will be said to be a heterogonal set in $\mathfrak{B}$ if

(i) $K^{-1} \leqq\left\|\psi_{\alpha}\right\| \leqq K$, for some $K$, for all $\alpha \varepsilon M$;

(ii) For every set of selectors $\left\{\gamma_{\alpha} ; \alpha \varepsilon M\right\}$ the manifolds $\mathfrak{M}$ and $\mathfrak{N}$ spanned by $\left\{\gamma_{\alpha} \psi_{\alpha} ; \alpha \varepsilon M\right\}$ and $\left\{\left(1-\gamma_{\alpha}\right) \psi_{\alpha} ; \alpha \varepsilon M\right\}$, respectively, generate a projection.

The projections just mentioned will be called the projections of the heterogonal set. If $P_{1}, P_{2}$ are projections of the set, then $P_{1} P_{2}=P_{2} P_{1}$ is also a projection of the set. If $P_{1} P_{2}=0$, then $P_{1}+P_{2}$ is a projection of the set. It is clear from (ii) above, that the $\psi_{a}$ span $\mathfrak{B}$ and that 
$\sum_{1}^{n} a_{\alpha_{i}} \psi_{\alpha_{i}}=0$ implies $a_{\alpha_{i}}=0, i=1, \cdots, n$. The projections of (ii) are studied in the lemma which follows.

Lemma. The projections of a heterogonal set $\left\{\psi_{\alpha} ; \alpha \varepsilon M\right\}$ in $\mathfrak{B}$ are uniformly bounded.

The contrary is assumed. Then $M$ is an infinite set. It will be shown that there exist projections $P_{i}$ and closed linear manifolds $\mathfrak{P}_{i}$, $i=1,2, \cdots$, the $\mathfrak{P}_{i}$ spanned by certain finite subsets of $\left\{\psi_{\alpha} ; \alpha \varepsilon M\right\}$, such that $\mathfrak{B}_{i} \cdot \mathfrak{B}_{j}=0, i \neq j, \mathfrak{M}_{i} \subset \mathfrak{B}_{i}$, and such that the bound of $P_{i}$ on $\mathfrak{P}_{i}$ exceeds $i K$, where $K>0$ is arbitrary. Let $\mathfrak{M}$ be the closed linear manifold spanned by the manifolds $\mathfrak{M}_{i}, i=1,2, \cdots$. Let $P$ be that projection of the heterogonal set whose range is $\mathfrak{M}$. Then $P=P_{i}$ on $\mathfrak{P}_{i}$; hence $|P|>i K, i=1,2, \cdots$. This is the desired contradiction.

It remains to indicate how the $P_{i}, \mathfrak{B}_{i}$ are determined. Let $P_{1}^{\prime}$ be any projection such that $\left|P_{1}^{\prime}\right|>K$. Let $\left\|P_{1}^{\prime} f\right\|>K\|f\| ;$ let $N_{1}, \psi_{\alpha_{i}}, a_{\alpha_{i}}$, $i=1, \cdots, N_{1}$, be so chosen (by Definition 2 (ii)) that if $g=\sum_{1}^{N_{1}} a_{\alpha_{i}} \psi_{\alpha_{i}}$, $\|f-g\|$ is small; thus $\left\|P_{1}^{\prime} g\right\|>K\|g\|$. The manifold $\mathfrak{P}_{1}$ is the manifold spanned by $\left\{\psi_{\alpha_{n}}\right\}, n \leqq N_{1}$. Let $P_{\Re_{1}}$ be the projection of the heterogonal set whose range is $\mathfrak{P}_{1}$. Then $P_{1}$ is defined by $P_{1}=P_{\mathfrak{B}_{1}} \cdot P_{1}^{\prime}$. Let $M_{1}$ be the set $M$ minus the elements $\alpha_{1}, \cdots, \alpha_{N_{1}}$. Let $\mathfrak{Q}_{1}$ be the manifold spanned by $\left\{\psi_{\alpha} ; \alpha \varepsilon M_{1}\right\}$. Now it may be seen that, as a consequence of the assumption introducing the proof, there exists a projection $P_{2}^{\prime}$ whose bound over $\mathfrak{Q}_{1}$ exceeds $2 K$. This results from the equation $P f=P \cdot P_{\mathfrak{B}_{1}} f+P\left(f-P_{\mathfrak{B}_{1}} f\right)$ valid for any projection $P$ of the heterogonal set. We may, as above, find an integer $N_{2}>N_{1}$, elements $\psi_{\alpha_{n}}, N_{1}<n \leqq N_{2}$, such that if $\mathfrak{B}_{2}$ designates the manifold spanned by $\left\{\psi_{\alpha_{n}} ; N_{1}<n \leqq N_{2}\right\}$, the bound of $P_{2}^{\prime}$ on $\Re_{2}$ exceeds $2 K$. The projection $P_{2}$ is then defined by $P_{2}=P_{\mathfrak{B}_{2}} \cdot P_{2}^{\prime}$ where $P_{\mathfrak{B}_{2}}$ is the projection of the heterogonal set whose range is $\mathfrak{P}_{2}$. The construction of $P_{i}, \Re_{i}, i>2$, is now simple.

We are now in a position to prove the following theorem.

TheOREM A. A set of vectors $\left\{\phi_{\alpha} ; \alpha \varepsilon M\right\}$ is a basis in $\mathscr{B}$ if and only if it is a heterogonal set in $\mathfrak{B}$.

It will be asssumed that the set $\left\{\phi_{\alpha} ; \alpha \varepsilon M\right\}$ is a basis. Let $\left\{\gamma_{\alpha} ; \alpha \varepsilon M\right\}$ be a given set of selectors. Let $f \varepsilon \mathfrak{B}, f=\sum_{\alpha \in M} a_{\alpha} \phi_{\alpha}$. Since this series converges unconditionally, $\dagger$ any subseries converges. Thus

* That is, $\mathfrak{B}_{i}$ and $\mathfrak{B}_{j}$ have only the element 0 in common for $i \neq j$.

$\dagger$ W. Orlicz, Studia Mathematica, vol. 1 (1929), pp. 241-255. A series is said to converge unconditionally if the property of convergence is independent of the order of summation. 


$$
f=g+h, \quad g=\sum_{\alpha \in M} \gamma_{\alpha} a_{\alpha} \phi_{\alpha}, \quad h=\sum_{\alpha \in M}\left(1-\gamma_{\alpha}\right) a_{\alpha} \phi_{\alpha} .
$$

It will be shown that the representation $f=g+h$ is unique. This will be accomplished if it is shown that if $\mathfrak{M}$ is the manifold spanned by $\left\{\gamma_{\alpha} \phi_{\alpha} ; \alpha \varepsilon M\right\}$ and $j=\sum_{\alpha \in M} b_{\alpha} \phi_{\alpha} \varepsilon \mathfrak{M}$, then $b_{\alpha}=0$ in case $\gamma_{\alpha}=0$. A sequence $\left\{\phi_{\alpha_{i}}\right\}$ is chosen in such a way as to permit writing $j=\sum_{1}^{\infty} b_{i} \phi_{\alpha_{i}}, \quad k=\sum_{1}^{N} \gamma_{\alpha_{i}} c_{i} \phi_{\alpha_{i}}, \quad l=\sum_{1}^{n} b_{i} \phi_{\alpha_{i}}-\sum_{1}^{N} \gamma_{\alpha_{i}} c_{i} \phi_{\alpha_{i}}=\sum d_{i} \phi_{\alpha_{i}}$, with $\|l\|$ small at will by suitable choice of $n, N, c_{i}$. If $\gamma_{\alpha_{i}}=0, b_{i} \neq 0$, then $d_{i}=b_{i}$. Now there exists a constant $L$ such that

$$
\left|d_{i}\right| \leqq L\|l\| /\left\|\phi_{\alpha_{i}}\right\| \leqq K L\|l\| .^{*}
$$

Thus $\gamma_{\alpha_{i}}=0$ implies $b_{i}=0$.

It is now assumed that $\left\{\phi_{\alpha} ; \alpha \varepsilon M\right\}$ is a heterogonal set. Let $\alpha$ be a fixed element in $M$; let $P_{\alpha}$ be that projection of the heterogonal set whose range is spanned by $\phi_{\alpha}$. The numbers $a_{\alpha}$ required in Definition 1 are defined by $P_{\alpha} f=a_{\alpha} \phi_{\alpha}$. Since $\left\{\phi_{\alpha} ; \alpha \varepsilon M\right\}$ spans $\mathfrak{B}$, for an $\epsilon>0$, elements $\phi_{\alpha_{i}}$ and numbers $b_{\alpha_{i}}$ can be found, $i=1, \cdots, N(\epsilon)$, such that

$$
\left\|f-\sum_{1}^{N} b_{\alpha_{i}} \phi_{\alpha_{i}}\right\|<\epsilon .
$$

This implies the existence of a denumerable set $M^{\prime} \subset M$ such that $\left\{\phi_{\alpha} ; \alpha \varepsilon M^{\prime}\right\}$ spans a manifold containing $f$. If $\alpha \leftrightarrow M^{\prime}$, then $P_{\alpha} f=0$. Thus all but at most a denumerable number of the "coordinates" $a_{\alpha}$ are zero. Let $\left\{\alpha_{i}\right\}, i=1,2, \cdots$, be any ordering of $M^{\prime}$. It will be shown that

$$
\lim _{n \rightarrow \infty}\left\|f-\sum_{1}^{n} a_{\alpha_{i}} \phi_{\alpha_{i}}\right\|=0
$$

Write $P_{n}=\sum_{1}^{n} P_{\alpha_{i}}$; for $\epsilon>0$, numbers $b_{i}, i=1, \cdots, N$, may be found such that

$$
\left\|f-\sum_{1}^{N} b_{i} \phi_{\alpha_{i}}\right\|<\epsilon .
$$

Choose $L>0$ by the Lemma such that $\left|P_{n}\right| \leqq L$. Then for $n \geqq N$,

Thus

$$
\left\|P_{n}\left(f-\sum_{1}^{N} b_{i} \phi_{\alpha_{i}}\right)\right\|=\left\|P_{n} f-\sum_{1}^{N} b_{i} \phi_{\alpha_{i}}\right\| \leqq L \epsilon .
$$

* S. Banach, loc. cit., p. 111. The proof may be altered to meet our requirements. 


$$
\left\|f-P_{n} f\right\|=\left\|f-\sum_{1}^{n} a_{\alpha_{i}} \phi_{\alpha_{i}}\right\| \leqq(1+L) \epsilon, \quad n \geqq N .
$$

Toward proving the unique representation of $f$, assume

$$
f=\sum_{\alpha \in M} a_{\alpha} \phi_{\alpha}=\sum_{\alpha \in M} c_{\alpha} \phi_{\alpha} .
$$

Then $0=\sum_{\alpha \in M}\left(a_{\alpha}-c_{\alpha}\right) \phi_{\alpha}$; instead of $\sum_{\alpha \in M}\left(a_{\alpha}-c_{\alpha}\right) \phi_{\alpha}$, write $\sum_{1}^{\infty} d_{i} \phi_{\alpha_{i}}$. Thus for $\epsilon>0, n>n_{0},\left\|\sum_{1}^{n} d_{i} \phi_{\alpha_{i}}\right\|<\epsilon$, and for $i \leqq n$,

$$
\left\|P_{\alpha_{i}}\left(\sum_{1}^{n} d_{i} \phi_{\alpha_{i}}\right)\right\|=\left\|d_{i} \phi_{\alpha_{i}}\right\| \leqq L \epsilon
$$

and $d_{i}=0$.

2. Bicontinuous transformations in $\leftleftarrows$. From now on, the space under consideration is a complex euclidean space $\mathbb{E}$. In such a space it is always possible to find a complete orthonormal set $\left\{\phi_{\alpha} ; \alpha \varepsilon M\right\}$ which is a basis for $\mathbb{E} ; f \varepsilon$ \& implies

$$
f=\sum_{\alpha \in M} a_{\alpha} \phi_{\alpha}, \quad\|f\|^{2}=\sum_{\alpha \in M}\left|a_{\alpha}\right|^{2} .
$$

THEOREM B. Let $\left\{\phi_{\alpha} ; \alpha \varepsilon M\right\}$ be a basis in a complex euclidean space (ङ. The set of vectors $\left\{\psi_{\alpha} ; \alpha \varepsilon M\right\}$ is a basis in \& if and only if there exists a bicontinuous transformation $T$ such that $T \phi_{\alpha}=\psi_{\alpha}, \alpha \varepsilon M$.

The existence of $T$ is assumed. Let

$$
K^{-1} \leqq\left\|\phi_{\alpha}\right\| \leqq K, \quad \alpha \varepsilon M ; \quad|T| \leqq S, \quad\left|T^{-1}\right| \leqq S .
$$

Then $(K S)^{-1} \leqq\left\|T \phi_{\alpha}\right\| \leqq K S$. If $f \varepsilon$ \&,

$$
f=T\left(T^{-1} f\right)=T\left(\sum_{\alpha \in M} a_{\alpha} \phi_{\alpha}\right)=\sum_{\alpha \in M} a_{\alpha}\left(T \phi_{\alpha}\right)
$$

gives the expansion of $f$ in terms of the $\psi_{\alpha}=T \phi_{\alpha}$. It can readily be seen that $\left\{\psi_{\alpha} ; \alpha \varepsilon M\right\}$ is a basis in $₹$.

It is now assumed that $\left\{\psi_{\alpha} ; \alpha \varepsilon M\right\}$ is a basis in $₹$. We may and shall assume that $\left\{\phi_{\alpha} ; \alpha \varepsilon M\right\}$ is a complete orthonormal set. Let $L>0$ be the uniform bound of the projections of the heterogonal set $\left\{\psi_{\alpha} ; \alpha \varepsilon M\right\}$ (the Lemma and Theorem A). Using the identity

$$
\|f+g\|^{2}+\|f-g\|^{2}=2\left(\|f\|^{2}+\|g\|^{2}\right),
$$


with $f$ and $g$ arbitrary in $\mathbb{E}$, which characterizes $\mathbb{E},^{*}$ one obtains for arbitrary $\psi_{\alpha_{i}}, a_{\alpha_{i}}, i=1, \cdots, n$,

$$
\begin{aligned}
2^{n} K^{-2} \sum_{1}^{n}\left|a_{\alpha_{i}}\right|^{2} & \leqq \sum^{\prime}\left\|\sum_{1}^{n} \pm a_{\alpha_{i}} \psi_{\alpha_{i}}\right\|^{2} \\
& =2^{n} \sum_{1}^{n}\left\|a_{\alpha_{i}} \psi_{\alpha_{i}}\right\|^{2} \leqq 2^{n} K^{2} \sum_{1}^{n}\left|a_{\alpha_{i}}\right|^{2},
\end{aligned}
$$

where $\sum^{\prime}$ is a sum extended over all possible sign distributions. This shows that for some particular sign distribution $\delta_{i}= \pm 1$,

Now

$$
\left\|\sum_{1}^{n} \delta_{i} a_{\alpha_{i}} \psi_{\alpha_{i}}\right\| \leqq K\left(\sum_{1}^{n}\left|a_{\alpha_{i}}\right|^{2}\right)^{1 / 2}
$$

$$
\sum_{1}^{n} a_{\alpha_{i}} \psi_{\alpha_{i}}=\sum_{1}^{n} \delta_{i} \frac{1+\delta_{i}}{2} a_{\alpha_{i}} \psi_{\alpha_{i}}-\sum_{1}^{n} \delta_{i} \frac{1-\delta_{i}}{2} a_{\alpha_{i}} \psi_{\alpha_{i}}
$$

and each of the last two vectors is a projection of $\sum_{1}^{n} \delta_{i} a_{\alpha_{i}} \psi_{\alpha_{i}}$. Thus

$$
\left\|\sum_{1}^{n} a_{\alpha_{i}} \psi_{\alpha_{i}}\right\| \leqq 2 K L\left(\sum_{1}^{n}\left|a_{\alpha_{i}}\right|^{2}\right)^{1 / 2} \text {. }
$$

This implies that the transformation which carries $\sum_{1}^{n} a_{\alpha_{i}} \phi_{\alpha_{i}}$ into $\sum_{1}^{n} a_{a_{i}} \psi_{\alpha_{i}}$ is continuous. The closed linear extension of this transformation will be called $T$.

The inequality (1) indicates that for some sign distribution $\delta_{i}^{\prime}= \pm 1$,

$$
\left\|\sum_{1}^{n} \delta_{i}^{\prime} a_{\alpha_{i}} \psi_{\alpha_{i}}\right\| \geqq K^{-1}\left(\sum_{1}^{n}\left|a_{\alpha_{i}}\right|^{2}\right)^{1 / 2} ;
$$

and since

$$
\sum_{1}^{n} \delta_{i}^{\prime} a_{\alpha_{i}} \psi_{\alpha_{i}}=\sum_{1}^{n} \frac{1+\delta_{i}^{\prime}}{2} a_{\alpha_{i}} \psi_{\alpha_{i}}-\sum_{1}^{n} \frac{1-\delta_{i}^{\prime}}{2} a_{\alpha_{i}} \psi_{\alpha_{i}},
$$

this gives

$$
\left\|\sum_{1}^{n} a_{\alpha_{i}} \phi_{\alpha_{i}}\right\| \leqq 2 K L\left\|\sum_{1}^{n} a_{\alpha_{i}} \psi_{\alpha_{i}}\right\| .
$$

This implies that the transformation $T$ has a bounded inverse.

\section{Barnard College, Columbia University}

* Jordan and von Neumann, Annals of Mathematics, (2), vol. 36 (1935), pp. 719723. 